\title{
A Comparison for the Simulation of Frictionless Contact Problem with Large Displacement
}

\author{
Z. M. Nizam ${ }^{1, a}$, H. Obiya ${ }^{2, b}$, K. ljima ${ }^{3, c}$ and A. T. S. Azhar ${ }^{4, d}$ \\ ${ }^{1 \& 4}$ University Tun Hussein Onn Malaysia, Malaysia \\ 2 \& 3 Saga University, Japan

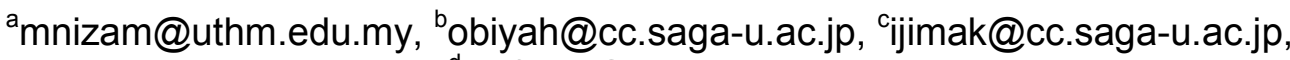 \\ dsaifulaz@uthm.edu.my
}

Keywords: Frictionless Contact Problem, Tangent Stiffness Method, Timoshenko Beam Theory.

\begin{abstract}
Studies of contact problem have been widely executed by researchers with variable scopes, methods and definitions. A common problem that occurs while handling contact phenomena is sliding through element boundary [1], due to the discontinuity of the local coordinate between elements and a contact point [2] [3]. This common problem that occurs at an element boundary is such that a stable convergence result is not easily obtained. [4], thus inspires authors to make a comparison of two beam methods which are Euler-Bernoulli beam theory and Timoshenko beam theory for frictionless contact problem. The authors have been investigate geometrically non-linear analysis with extremely large displacement by the aid of Tangent Stiffness Method (TSM) [5], a robust non-linear analysis method to execute analysis and produce results with high accuracy. In this study, the authors propose the modification of beam elements with three nodes by considering the adaptation of shear deformation by Timoshenko beam theory. The modification enables the contact point to slide through the element edge smoothly and some numerical examples are provided in this study.
\end{abstract}

\section{Tangent Stiffness Method}

The TSM was solely idealized to overcome numerical cases exhibiting significant nonlinearity. The superiority of this method is that it converges the unbalanced force with high accuracy by defining element behavior using a simple form of the element force equation. This theory requires the element edge forces to be treated separately and independently of each other. In addition, strict compatibility and an equilibrium equation are disseminated in the iteration configuration to converge the unbalanced force.

Here, let an element constituted by two edges with its element edge forces and the force vector for both edges is assumed as $\mathbf{S}$. Let the external force vector as $\mathbf{U}$, in a plane coordinate system with $\mathbf{J}$ as the equilibrium matrix, and the equilibrium condition could be expressed as the following equation.

$$
\mathbf{U}=\mathbf{J S}
$$

With the differentiation of Eq. (1), the tangent stiffness equation could be expressed as;

$$
\boldsymbol{\delta} \mathbf{U}=\boldsymbol{\delta} \mathbf{J}+\mathbf{J} \boldsymbol{\delta} \mathbf{S}=\left(\mathbf{K}_{\mathbf{0}}+\mathbf{K}_{\mathbf{G}}\right) \delta \mathbf{d}
$$

Here, the differentiation of Eq. (1) simultaneously extract $\delta \mathbf{S}$ and $\delta \mathbf{J}$ makes it possible to express a linear function of displacement vector, $\delta \mathbf{d}$ in the local coordinate system. Meanwhile, in Eq. (2), $\mathbf{K}_{\mathbf{O}}$ represents the element stiffness matrix which also simulates the element behavior, correspondent to the element stiffness in the coordinate system while $\mathbf{K}_{\mathbf{G}}$, represents the element displacement originated by the tangent geometrical stiffness. 


\section{Comparison of Euler-Bernoulli and Timoshenko beam theory for extremely large loading increment}

With the aforementioned method, TSM could solve any geometrically non-linear problem, even for extremely large deformation. Therefore, in this section, author will provide a comparison for extremely large loading for both Euler-Bernoulli beam and Timoshenko beam with a common plane frame structure.

$$
\left[\begin{array}{c}
N \\
M_{i} \\
M_{j}
\end{array}\right]=\left[\begin{array}{ccc}
\frac{\mathrm{EA}}{l_{0}} & 0 & 0 \\
0 & \frac{4 \mathrm{EI}}{l_{0}} & \frac{2 \mathrm{EI}}{l_{0}} \\
0 & \frac{2 \mathrm{EI}}{l_{0}} & \frac{4 \mathrm{EI}}{l_{0}}
\end{array}\right]\left[\begin{array}{c}
\delta l \\
\theta_{i} \\
\theta_{j}
\end{array}\right]
$$

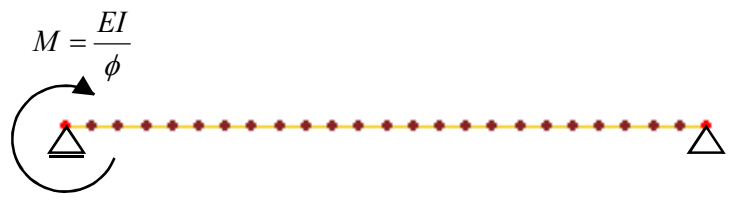

Figure 1 Plane beam in pre-loading state

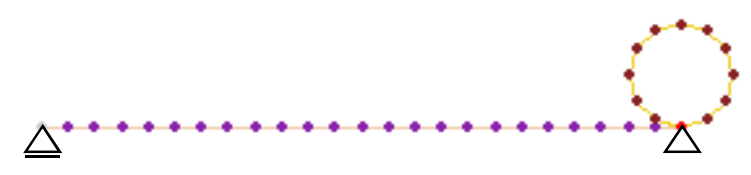

Figure 2 Plane beam in post-loading state

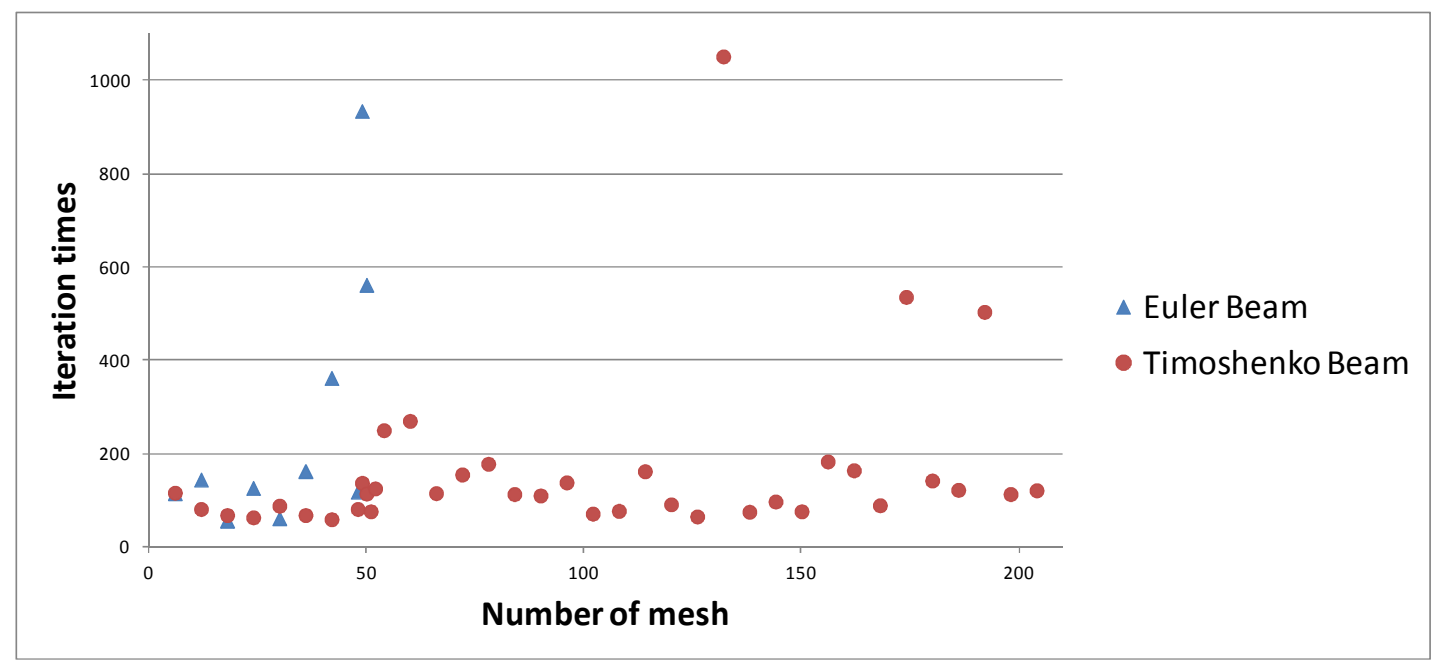

Figure 3 The relation between number of mesh vs. iteration times

Eq. (3) represents a common element force equation for Euler-Bernoulli beam, while Eq. (4) shows Timoshenko beam theory with the consideration of shear deformation, shown in Eq. (5). Meanwhile, Fig. (1) shows a simply supported beam with a roller support at one end and a pinned support at the other. An extremely large bending moment is applied in a single incremental step at the roller support until the beam deformed to a circular shape. In addition, as shown in Fig. 3, the beam 
meshes are set from 6 to 200 meshes, and for this case, a stable convergence result for Euler-Bernoulli beam is until 52 meshes, while for Timoshenko beam, stable convergence result has been achieved even until 200 meshes.

\section{Comparison of Euler-Bernoulli and Timoshenko beam theory for frictionless contact problem}

In this section, the element force equation for Euler-Bernoulli and Timoshenko beam is introduced. Fig. (4) shows an equilibrium condition of an elastic and homogeneous simply supported beam which is subjected by an axial force $N$, edge moments $M_{i}$ and $M_{j}$, and contact force $Y_{c}$. The element force equation for Euler-Bernoulli beam in contact case is shown in Eq. (6) and for Timoshenko beam is shown in Eqs. (7), (8) and (9). The difference between these two theories are in Timoshenko beam, shear deformation $(\gamma)$ is considered even for the large deformational case.
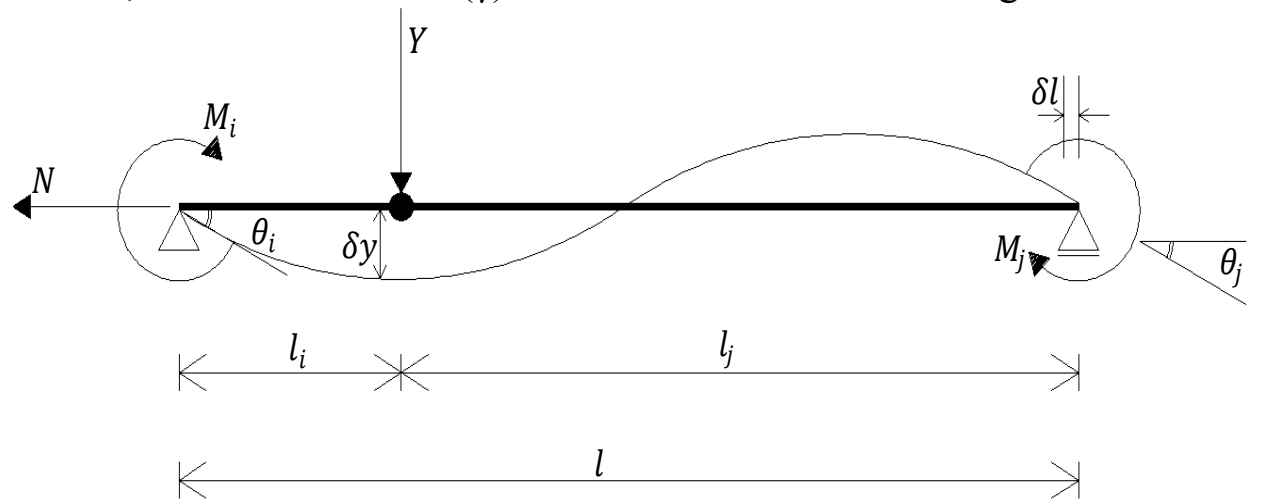

Figure 4 Contact problem for a simply supported beam coordinate

$$
\begin{aligned}
& {\left[\begin{array}{c}
N \\
M_{i} \\
M_{j} \\
Y
\end{array}\right]=\left\{\frac{\mathrm{EI}}{l_{0}}\left[\begin{array}{cccc}
0 & 0 & 0 & 0 \\
0 & \frac{4 l_{i c}+3 l_{j c}}{l_{i c}} & \frac{9 l_{0}{ }^{2}-l_{i c}{ }^{2} l_{j c}{ }^{2}}{l_{i c}{ }^{2} l_{j c}{ }^{2}} & -\frac{3 l_{j c} l_{0}{ }^{2}}{l_{i c}{ }^{2}{ }_{j c}{ }^{2}} \\
0 & \frac{9 l_{0}{ }^{2}-l_{i c}{ }^{2} l_{j c}{ }^{2}}{l_{i c}{ }^{2}{ }^{2}{ }^{2}} & \frac{4 l_{j c}+3 l_{i c}}{l_{j c}} & \frac{3 l_{i c} l_{0}{ }^{2}}{l_{i c}{ }^{2}{ }^{2}} \\
0 & -\frac{3 l_{j c} l_{0}{ }^{2}}{l_{i c}{ }^{2}{ }^{2}} & \frac{3 l_{i c} l_{0}{ }^{2}}{l_{i c}{ }^{2}{ }^{2}{ }^{2}} & \frac{3 l_{0}{ }^{3}}{l_{i c}{ }^{3}{ }^{3}}
\end{array}\right]+\left[\begin{array}{cccc}
\frac{\mathrm{EA}}{l_{0}} & 0 & 0 & 0 \\
0 & 0 & 0 & 0 \\
0 & 0 & 0 & 0 \\
0 & 0 & 0 & 0
\end{array}\right]\right\}\left[\begin{array}{c}
\delta l \\
\theta_{i} \\
\theta_{j} \\
\delta y_{c}
\end{array}\right]}
\end{aligned}
$$

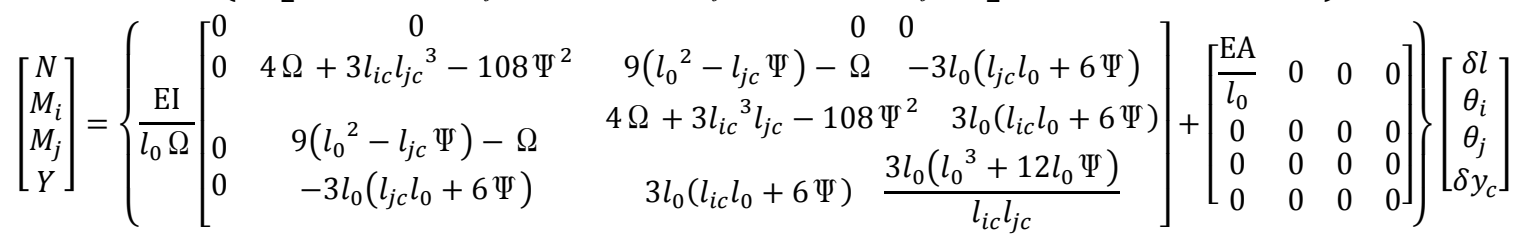

$$
\begin{aligned}
& \Psi=\frac{\mathrm{EI}}{\mathrm{GA}} \\
& \Omega=\left(l_{i c} l_{j c}\right)^{2}+3 \Psi\left(l_{0}{ }^{2}+l_{i c} l_{j c}\right)+36 \Psi^{2}
\end{aligned}
$$

\section{Numerical example}

In this analysis, a cantilever beam configuration as shown in Fig. (5) is applied for contact analysis. The beam is consisted by 18 segments and 19 nodes. As shown in Fig. (6) (a) to (f), a compulsory displacement in upwards direction is applied on a contact node, which is marked with the red node. In this analysis, we will investigate the territory which leads to divergence of the unbalanced force. Fig. (6) shows the relation between percentage of $l_{i} / l$ in a single contact element 
and the displacement of contact node in post-contact condition. The contact node position is set in six different positions, which are $4.05 \mathrm{~m}, 4.1 \mathrm{~m}, 4.2 \mathrm{~m}, 4.3 \mathrm{~m}, 4.35 \mathrm{~m}$ and $4.4 \mathrm{~m}$ in horizontal direction.

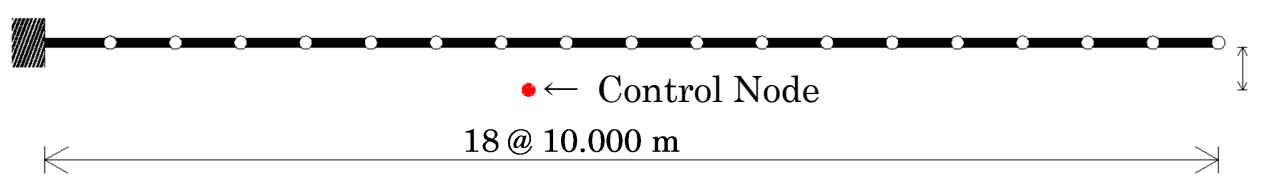

Figure 5 Cantilever beam model

From the analysis result in Fig. (7), it is significantly clear that by the consideration of shear deformation in Timoshenko beam, a stable yet converged solution have been successfully achieved at the edge of the segment which ranges from $99.499 \%$ to $99.933 \%$. For Euler beam, the percentage ranges from $87.408 \%$ to $92.251 \%$ and for cantilever coordinate, it ranges from $96.135 \%$ to $97.836 \%$.

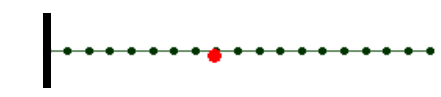

(a)

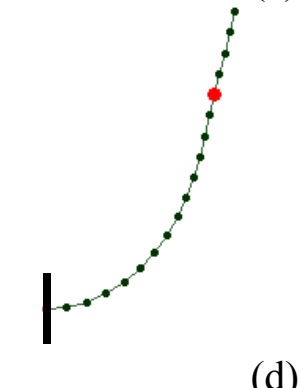

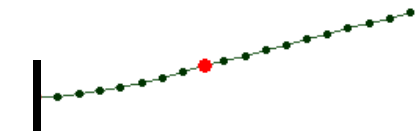

(b)

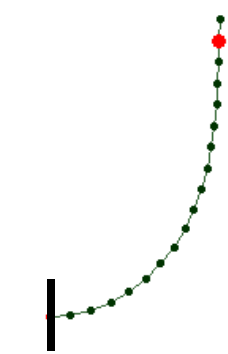

(e)

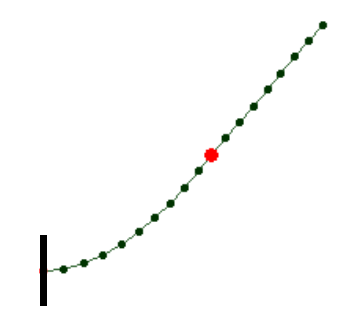

(c)

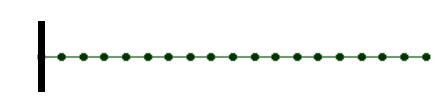

(f)

Figure 6 The cantilever beam deformation due to the contact node

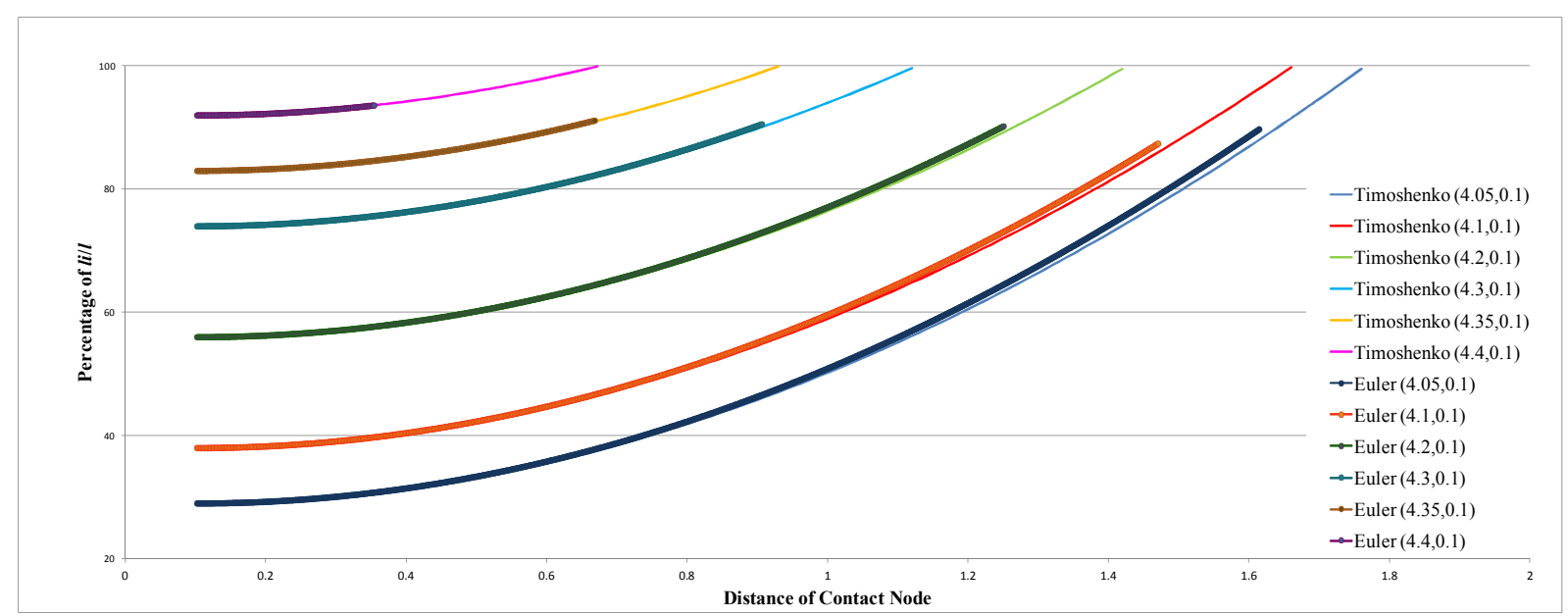

Figure 7 The relation between percentage $l_{i} / l$ and the distance of the contact node

\section{Summary}

For the Euler-Bernoulli beam theory, unbalanced force will either diverge or no convergence result could be achieved beyond the ranges. On the other hand, for Timoshenko beam, unbalanced force is steadily converged around the tip of the segment, and the contact node is able to slide through to the next segment. 


\section{References}

[1] X. Chen, K. Nakamura, M. Mori, T. Hisada, Finite element analysis for large deformation frictional contact problems with finite sliding, JSME International Journal. 64 (1998) 50-57.

[2] T. Tsutsui, H. Obiya, K. Ijima, An algorithm for contact problem with large deformation of plane frame structures, Advances in Computational Engineering \& Science. (2009).

[3] Z. M. Nizam, H. Obiya, A study on non-friction contact problem with large deformational analyses, Malaysian Technical Universities Conference on Engineering and Technology. (2008).

[4] A. Konyukov, Geometrically exact covariant approach for contact between curves, Computer Methods in Applied Mechanics and Engineering. 199 (2010) 2510-2531.

[5] H. Obiya, A study on accuracy and versatile of the tangent stiffness method by separation of element stiffness from geometrical stiffness (In Japanese), Saga University. (1998). 